\title{
Orthogonal stability of the generalized quadratic functional equations in the sense of Rätz
}

https://doi.org/10.1515/dema-2019-0044

Received February 13, 2019; accepted November 14, 2019

Abstract: Let $(X, \perp)$ be an orthogonality module in the sense of Rätz over a unital Banach algebra $A$ and $Y$ be a real Banach module over $A$. In this paper, we apply the alternative fixed point theorem for proving the Hyers-Ulam stability of the orthogonally generalized $k$-quadratic functional equation of the form

$$
a f(k x+y)+a f(k x-y)=f(a x+a y)+f(a x-a y)+\left(2 k^{2}-2\right) f(a x)
$$

for some $|k|>1$, for all $a \in A_{1}:=\{u \in A \mid\|u\|=1\}$ and for all $x, y \in X$ with $x \perp y$, where $f$ maps from $X$ to $Y$.

Keywords: fixed point method, orthogonally generalized quadratic functional equation, stability

MSC: Primary 39B52, 39B55, 39B72; Secondary 47H10, 46H25

\section{Preliminaries and introduction}

The orthogonality relation has various definitions in normed spaces such as Birkhoff orthogonality, isosceles orthogonality and Pythagorean orthogonality. In this paper, we study the orthogonal stability of the functional equations in the sense of Rätz as follows:

Definition 1.1. [1] Let $X$ be a real vector space with $\operatorname{dim}(X) \geq 2$, and $\perp$ is a binary relation on $X$ satisfying the following properties:

1. $x \perp 0$ and $0 \perp x$ for all $x \in X$;

2. if $x, y \in X \backslash\{0\}$ and $x \perp y$, then then $x, y$ are linearly independent;

3. if $x, y \in X$ and $x \perp y$, then $\alpha x \perp \beta y$ for all $\alpha, \beta \in \mathbb{R}$;

4. for any two-dimensional subspace $P$ of $X$, and for every $x \in P, \lambda \in[0, \infty)$, there exists $y_{0} \in P$ such that $x \perp y_{0}$ and $x+y_{0} \perp \lambda x-y_{0}$.

An ordered pair $(X, \perp)$ is called an orthogonality space in the sense of Rätz, or shortly, orthogonality space.

Some interesting examples of orthogonality spaces are as follows:

- the trivial orthogonality on a vector space $X$ defined by $x \perp 0$ and $0 \perp x$ for all $x \in X$;

- for any non-zero elements $x, y \in X$, we define $\perp$ by $x \perp y$ if and only if $x, y$ are linearly independent;

- the ordinary orthogonality on an inner product space $(X,\langle\cdot, \cdot\rangle)$ given by $x \perp y$ if and only if $\langle x, y\rangle=0$;

- the Birkhoff-James orthogonality on a normed space $(X,\|\cdot\|)$ defined by $x \perp y$ if and only if $\|x+\lambda y\| \geq$ $\|x\|$ for all $\lambda \in \mathbb{R}$.

Laddawan Aiemsomboon: Department of Mathematics and Statistics, Faculty of Science and Technology, Thammasat University Rangsit Center, Pathum Thani 12120, Thailand; E-mail: Laddawan_Aiemsomboon@hotmail.com

${ }^{\star}$ Corresponding Author: Wutiphol Sintunavarat: Department of Mathematics and Statistics, Faculty of Science and Technology, Thammasat University Rangsit Center, Pathum Thani 12120, Thailand; E-mail: wutiphol@mathstat.sci.tu.ac.th 
The problem of stability of functional equations was first initialed by a question of Ulam [2]. Afterward, Hyers [3] gave partial answer of Ulam's question in the context of Banach spaces. The result of Hyers [3] was extended by Aoki [4], Bourgin [5], and Forti [6]. In 1978, Rassias [7] attempted to weaken the condition for the bound of the norm of Cauchy difference and proved a considerably generalized Hyers' result in [3] by using of a directed method as follows:

Theorem 1.2. [7] Let $E_{1}$ and $E_{2}$ be two Banach spaces and $f: E_{1} \rightarrow E_{2}$ be a function. Iff satisfies the following inequality

$$
\|f(x+y)-f(x)-f(y)\| \leq \delta\left(\|x\|^{p}+\|y\|^{p}\right)
$$

for some $\delta \geq 0, p$ with $0 \leq p<1$ and for all $x, y \in E_{1}$, then there exists a unique additive function $g: E_{1} \rightarrow E_{2}$ such that

$$
\|f(x)-g(x)\| \leq \frac{2 \delta}{2-2^{p}}\|x\|^{p}
$$

for each $x \in E_{1}$. If, in addition, $f(t x)$ is continuous in $t$ for each fixed $x \in E_{1}$, then the function $g$ is linear.

Remark 1.3. If $p=0$, then Theorem 1.2 reduces to Hyers' result in [3].

In 1991, Gajda [8] gave the above result for $p>1$ and gave an example showing that an analogous result for $p=1$ is not true. Afterward, Rassias $[9,10]$ motivated by Theorem 1.2 proved the following generalization:

Theorem 1.4. $[9,10]$ Let $E_{1}$ be a normed space, $E_{2}$ be a Banach space and $f: E_{1} \rightarrow E_{2}$ be a function. If $f$ satisfies the following inequality

$$
\|f(x+y)-f(x)-f(y)\| \leq \delta\left(\|x\|^{p}+\|y\|^{p}\right)
$$

for some $\delta \geq 0, p \neq 1$ and for all $x, y \in E_{1}-\left\{0_{E_{1}}\right\}$, then there exists a unique additive function $g: E_{1} \rightarrow E_{2}$ such that

for each $x \in E_{1}-\left\{0_{E_{1}}\right\}$

$$
\|f(x)-g(x)\| \leq \frac{2 \delta}{\left|2-2^{p}\right|}\|x\|^{p}
$$

Next, we give some of the results present in the literature concerning stability and orthogonally stability results. In 1995, Ger and Sikorska [11] showed the orthogonally stability result of the Cauchy functional equation as follow:

Theorem 1.5. [11] Let $(X, \perp)$ be an orthogonality space, $Y$ be a real Banach space and $f: X \rightarrow Y$ be a function. If $f$ satisfies the following inequality

$$
\|f(x+y)-f(x)-f(y)\| \leq \varepsilon
$$

for some $\varepsilon>0$ and for all $x, y \in X$ with $x \perp y$, then there exists exactly one orthogonally additive mapping $g: X \rightarrow Y$ such that

for all $x \in X$.

$$
\|f(x)-g(x)\| \leq \frac{16}{3} \varepsilon
$$

On the other hand, Skof [12] first sutied the stability of the quadratic functional equation and proved the following theorem:

Theorem 1.6. [12] Let $(X, \perp)$ be an orthogonality space, $Y$ be a real Banach space and $f: X \rightarrow Y$ be a mapping. If $f$ satisfies the following inequality

$$
\|f(x+y)+f(x-y)-2 f(x)-2 f(y)\| \leq \varepsilon
$$

for some $\varepsilon>0$ and for all $x, y \in X$, then there is a unique quadratic mapping $g: X \rightarrow Y$, i.e. it satisfies the following functional equation:

$$
g(x+y)+g(x-y)=2 g(x)+2 g(y)
$$


for all $x, y \in X$ such that

$$
\|f(x)-g(x)\| \leq \frac{\varepsilon}{2}
$$

for all $x \in X$.

Afterward, Vajzović [13] considered the orthogonal quadratic functional equation

$$
f(x+y)+f(x-y)=2 f(x)+2 f(x)
$$

for all $x, y \in X$ with $x \perp y$ where $f$ is a continuous mapping from a Hilbert space $X$ into a scalar field $Y$ and $\perp$ means the Hilbert space orthogonality.

In 2008, Towanlong and Nakmahachalasint [14] investigated the Hyers-Ulam Rassias stability of the quadratic functional equation

$$
f(3 x+y)+f(3 x-y)=f(x+y)+f(x-y)+16 f(x)
$$

for all $x, y \in X$, where $f$ maps from a real vector space $X$ to a real Banach space $Y$.

The purpose of this work is to prove the orthogonal stability of the generalized quadratic functional equation of the form

$$
a f(k x+y)+a f(k x-y)=f(a x+a y)+f(a x-a y)+\left(2 k^{2}-2\right) f(a x)
$$

for some $|k|>1$, for all $a \in A_{1}:=\{u \in A \mid\|u\|=1\}$ and all $x, y \in X$ with $x \perp y$ in the sense of Rätz, where $f$ maps from an orthogonality module $(X, \perp)$ over a unital Banach algebra $A$ to a real Banach module $Y$ over $A$.

\section{Main result}

Now, we recall the classical fundamental result from the metric fixed point theory which is the main tool of the proof used in the main result. We refer to [15] for the proof of this result.

Theorem 2.1. [15] Let $(X, d)$ be a complete generalized metric space and $J: X \rightarrow X$ be a contraction mapping with the contractive constant $L<1$, that is, $d(J x, J y) \leq L d(x, y)$ for all $x, y \in X$. Then for each given element $x \in X$, either

$$
d\left(J^{n} x, J^{n+1} x\right)=\infty
$$

for all nonnegative integers $n$ or there exists a positive integer $n_{0}$ such that

1. $d\left(J^{n} x, J^{n+1} x\right)<\infty$ for all $n \geq n_{0}$;

2. J has a unique fixed point $y^{\star}$ in the set

$$
Y=\left\{y \in X: d\left(J^{n_{0}} x, y\right)<\infty\right\} ;
$$

3. the sequence $\left\{J^{n} x\right\}$ converges to a fixed point $y^{\star}$ of $J$;

4. $d\left(y, y^{\star}\right) \leq \frac{1}{1-L} d(y, J y), \forall y \in Y$.

Let $X$ be an orthogonality module over a unital Banach algebra $A$ with the unit $e, Y$ be a real Banach module over $A$ and $A_{1}:=\{u \in A \mid\|u\|=1\}$. Throughout this work, for a given mapping $f: X \rightarrow Y,|k|>1, a \in A_{1}$ and $x, y \in X$, we will use the following symbol:

$$
D_{a}^{k} f(x, y):=a f(k x+y)+a f(k x-y)-f(a x+a y)-f(a x-a y)-\left(2 k^{2}-2\right) f(a x) .
$$

For $|k|>1$, a mapping $f: X \rightarrow Y$ is said to be orthogonal generalized $k$-quadratic if it satisfies the equation

$$
f(k x+y)+f(k x-y)=f(x+y)+f(x-y)+\left(2 k^{2}-2\right) f(x)
$$

for all $x, y \in X$ with $x \perp y$. 
Definition 2.2. [16] Let $X$ be an orthogonality module over a unital Banach algebra $A$ with the unit $e, Y$ be a real Banach module over $A$. An additive mapping $f: X \rightarrow Y$ is called an A-additive mapping if

$$
f(a x)=a f(x)
$$

for all $x \in X$ and for all $a \in A$.

Next, we give the main theorem about stability of the generalized quadratic functional equations in the sense of Rätz.

Theorem 2.3. Let $(X, \perp)$ be an orthogonality module over a unital Banach algebra $A$ with the unit $e, Y$ be $a$ real Banach module over $A$ and $\rho: X \times X \rightarrow[0, \infty)$ be a function such that there exist real numbers $L, k$ with $0 \leq L<1$ and $|k|>1$ such that

$$
\rho(x, y) \leq k^{2} L \rho\left(\frac{x}{k}, \frac{y}{k}\right)
$$

for all $x, y \in X$. Suppose that $f: X \rightarrow Y$ is a mapping satisfying $f(0)=0$ and

$$
\left\|D_{a}^{k} f(x, y)\right\| \leq \rho(x, y)
$$

for all $a \in A_{1}$ and $x, y \in X$ with $x \perp y$. Then there exists a unique A-additive mapping $g: X \rightarrow Y$ satisfying the $k$-orthogonal quadratic functional equation (2.2) and

$$
\|f(x)-g(x)\| \leq \frac{1}{2 k^{2}-2 k^{2} L} \rho(x, 0)
$$

for all $x \in X$.

Proof. Let $V$ be the class of all mappings from $X$ into $Y$ and let the generalized metric $d$ on $V$ be defined by

$$
d(u, v)=\inf \{\beta>0:\|u(x)-v(x)\| \leq \beta \rho(x, 0), \forall x \in X\},
$$

where $\inf \emptyset=\infty$. Then $(V, d)$ is complete.

Next, we define the linear mapping $J: V \rightarrow V$ by

$$
(J u)(x):=\frac{1}{k^{2}} u(k x)
$$

for all $u \in V$ and $x \in X$. First, we claim that $J$ is a contraction mapping. Note that for each $u, v \in X$,

$$
\begin{aligned}
d(u, v) \leq \beta & \Rightarrow\|u(x)-v(x)\| \leq \beta \rho(x, 0), \quad \forall x \in X \\
& \Rightarrow\left\|\frac{1}{k^{2}} u(k x)-\frac{1}{k^{2}} v(k x)\right\| \leq \frac{1}{k^{2}} \beta \rho(k x, 0), \quad \forall x \in X \\
& \Rightarrow\|(J u)(x)-(J v)(x)\| \leq \beta L \rho(x, 0), \quad \forall x \in X \\
& \Rightarrow d(J u, J v) \leq \beta L .
\end{aligned}
$$

It follows that

$$
d(J u, J v) \leq L d(u, v)
$$

for all $u, v \in V$. Putting $y=0$ and $a=e$ in (2.4), we obtain

$$
\left\|2 f(k x)-2 k^{2} f(x)\right\| \leq \rho(x, 0)
$$

and hence

$$
\left\|f(x)-\frac{1}{k^{2}} f(k x)\right\| \leq \frac{1}{2 k^{2}} \rho(x, 0)
$$

for all $x \in X$. It follows from (2.9) that

$$
d(f, J f) \leq \frac{1}{2 k^{2}}<\infty
$$


By Theorem 2.1, there exist a mapping $g: X \rightarrow Y$ satisfying the following conditions:

- $g$ is a unique fixed point of $J$, that is,

$$
g(k x)=k^{2} g(x)
$$

for all $x \in X$. Also, the mapping $g$ belong to the set

$$
P:=\{v \in V \mid d(f, v)<\infty\} .
$$

This implies that $g$ is a unique mapping satisfying (2.10) such that there exists a $\beta \in(0, \infty)$ which satisfies

$$
\|f(x)-g(x)\| \leq \beta \rho(x, 0)
$$

for all $x \in X$.

- $d\left(J^{n} f, g\right) \rightarrow 0$ as $n \rightarrow \infty$. This implies that

$$
\lim _{n \rightarrow \infty} \frac{1}{k^{2 n}} f\left(k^{n} x\right)=g(x)
$$

for all $x \in X$.

$-d(f, g) \leq \frac{1}{1-L} d(f, J f)$, which implies the inequality

$$
d(f, g) \leq \frac{1}{2 k^{2}-2 k^{2} L}
$$

for all $x \in X$.

Putting $a=e$ in (2.4), it follows from (2.3), (2.4) and (2.11) that

$$
\begin{aligned}
\left\|g(k x+y)+g(k x-y)-g(x+y)-g(x-y)-\left(2 k^{2}-2\right) g(x)\right\| & =\lim _{n \rightarrow \infty} \frac{1}{k^{2 n}}\left\|D_{e}^{k} f\left(k^{n} x, k^{n} y\right)\right\| \\
& \leq \lim _{n \rightarrow \infty} \frac{1}{k^{2 n}} \rho\left(k^{n} x, k^{n} y\right) \\
& \leq \lim _{n \rightarrow \infty} L^{n} \rho(x, y) \\
& =0
\end{aligned}
$$

for all $x, y \in X$ with $x \perp y$. This implies that

$$
g(k x+y)+g(k x-y)-g(x+y)-g(x-y)-\left(2 k^{2}-2\right) g(x)=0
$$

for all $x, y \in X$ with $x \perp y$, that is,

$$
g(k x+y)+g(k x-y)=g(x+y)+g(x-y)+\left(2 k^{2}-2\right) g(x)
$$

for all $x, y \in X$ with $x \perp y$. Thus $g: X \rightarrow Y$ is a unique orthogonally $k$-quadratic additive mapping.

Putting $y=0$ in (2.4), it follows from (2.3), (2.4) and (2.11) that

$$
\begin{aligned}
\left\|2 a g(k x)-\left(2 k^{2}\right) g(a x)\right\| & =\lim _{n \rightarrow \infty} \frac{1}{k^{2 n}}\left\|D_{a}^{k} f\left(k^{n} x, 0\right)\right\| \\
& \leq \lim _{n \rightarrow \infty} \frac{1}{k^{2 n}} \rho\left(k^{n} x, 0\right) \\
& \leq \lim _{n \rightarrow \infty} L^{n} \rho(x, 0) \\
& =0
\end{aligned}
$$

for all $a \in A_{1}$ and for all $x \in X$. Then

$$
2 a g(k x)-2 k^{2} g(a x)=0
$$

for all $a \in A_{1}$ and for all $x \in X$, and thus

$$
g(a x)=\frac{a}{k^{2}} g(k x)=a g(x)
$$


for all $a \in A_{1}$ and $x \in X$. For each $a \in A$ with $a \neq 0$, we have

$$
g(a x)=g\left(\|a\| \frac{a}{\|a\|} x\right)=\|a\| g\left(\frac{a}{\|a\|} x\right)=\|a\| \frac{a}{\|a\|} g(x)=\operatorname{ag}(x)
$$

for all $x \in X$. Since $f(0)=0$, we get $A(0)=0$. Then $g: X \rightarrow Y$ is a unique $A$-additive mapping satisfying the orthogonal generalized $k$-quadratic functional equation. From (2.12), we have (2.5) holds. This complete the proof.

Corollary 2.4. Let $X$ be an orthogonality module over a unital Banach algebra $A$ with the unit $e, Y$ be a real Banach module over $A, c>0$ and $0 \leq p<2$. Suppose that $f: X \rightarrow Y$ is a mapping satisfying $f(0)=0$ and there exists a real number $k$ with $|k|>1$ such that

$$
\left\|D_{a}^{k} f(x, y)\right\| \leq c\left(\|x\|^{p}+\|y\|^{p}\right)
$$

for all $a \in A_{1}$ and for all $x, y \in X$ with $x \perp y$. Then there exists a unique A-additive mapping $g: X \rightarrow Y$ satisfying the $k$-orthogonal generalized quadratic functional equation (2.2) and

$$
\|f(x)-g(x)\| \leq \frac{c}{2 k^{2}-2|k|^{p}}\|x\|^{p}
$$

for all $x \in X$.

Proof. Letting $\rho(x, y)=c\left(\|x\|^{p}+\|y\|^{p}\right)$ for all $x, y \in X$ and choose $L=|k|^{p-2}<1$ in Theorem 2.3. Then we obtain this result.

Corollary 2.5. Let $X$ be an orthogonality module over a unital Banach algebra $A$ with the unit $e, Y$ be a real Banach module over $A, c>0, p, q \geq 0$ with $p+q<2$. Suppose that $f: X \rightarrow Y$ is a mapping satisfying $f(0)=0$ and there exists a real number $k$ with $|k|>1$ such that

$$
\left\|D_{a}^{k} f(x, y)\right\| \leq c\|x\|^{p}\|y\|^{q}
$$

for all $a \in A_{1}$ and for all $x, y \in X$ with $x \perp y$. Then there exists a unique A-additive mapping $g: X \rightarrow Y$ satisfying the $k$-orthogonal generalized quadratic functional equation (2.2) and

$$
\|f(x)-g(x)\| \leq \frac{c}{2 k^{2}-2|k|^{p+q}}\|x\|^{p}
$$

for all $x \in X$

Proof. Letting $\rho(x, y)=c\|x\|^{p}\|y\|^{q}$ for all $x, y \in X$ and choose $L=|k|^{p+q-2}<1$ in Theorem 2.3. Then we obtain this result.

Corollary 2.6. Let $X$ be an orthogonality module over a unital Banach algebra $A$ with the unit $e, Y$ be a real Banach module over $A, c_{1}, c_{2}>0$ and $0 \leq p<2$. Suppose that $f: X \rightarrow Y$ is a mapping satisfying $f(0)=0$ and there exists a real number $k$ with $|k|>1$ such that

$$
\left\|D_{a}^{k} f(x, y)\right\| \leq c_{1}\left(\|x\|^{p}+\|y\|^{p}\right)+c_{2}\|x\|^{\frac{p}{2}}\|y\|^{\frac{p}{2}}
$$

for all $a \in A_{1}$ and for all $x, y \in X$ with $x \perp y$. Then there exists a unique A-additive mapping $g: X \rightarrow Y$ satisfying the $k$-orthogonal generalized quadratic functional equation (2.2) and

$$
\|f(x)-g(x)\| \leq \frac{c_{1}+c_{2}}{2 k^{2}-2|k|^{p}}\|x\|^{p}
$$

for all $x \in X$.

Proof. Letting $\rho(x, y)=c_{1}\left(\|x\|^{p}+\|y\|^{p}\right)+c_{2}\|x\|^{\frac{p}{2}}\|y\|^{\frac{p}{2}}$ for all $x, y \in X$ and choose $L=|k|^{p-2}<1$ in Theorem 2.3. Then we obtain this result. 
In the same way of the proof of Theorem 2.3, we can prove the following results:

Theorem 2.7. Let $X$ be an orthogonality module over a unital Banach algebra $A$ with the unit $e, Y$ be a real Banach module over $A$, and $a \in A$. Let $\rho: X \times X \rightarrow[0, \infty)$ be a function such that there exist a nonnegative real number $L, k$ with $0 \leq L<1$ and $|k|>1$ such that

$$
\rho(x, y) \leq \frac{L}{k^{2}} \rho(k x, k y)
$$

for all $x, y \in X$. Suppose that $f: X \rightarrow Y$ is a mapping satisfying $f(0)=0$ and

$$
\left\|D_{a}^{k} f(x, y)\right\| \leq \rho(x, y)
$$

for all $a \in A_{1}$ and $x, y \in X$ with $x \perp y$. Then there exists a unique A-additive mapping $g: X \rightarrow Y$ satisfying the $k$-orthogonal quadratic functional equation (2.2) and

$$
\|f(x)-g(x)\| \leq \frac{L}{2 k^{2}-2 k^{2} L} \rho(x, 0)
$$

for all $x \in X$.

Proof. Let $(V, d)$ be the generalized metric space defined in the proof of Theorem 2.3. Now we consider the linear mapping $J: V \rightarrow V$ such that

$$
(J u)(x):=k^{2} u\left(\frac{x}{k}\right)
$$

for all $u \in V$ and for all $x \in X$. The remain of the proof is similar to the proof of Theorem 2.3.

Corollary 2.8. Let $X$ be an orthogonality module over a unital Banach algebra $A$ with the unit $e, Y$ be a real Banach module over $A, c>0$, and $p>2$. Suppose that $f: X \rightarrow Y$ is a mapping satisfying $f(0)=0$ and there exists a real number $k$ with $|k|>1$ such that

$$
\left\|D_{a}^{k} f(x, y)\right\| \leq c\left(\|x\|^{p}+\|y\|^{p}\right)
$$

for all $a \in A_{1}$ and for all $x, y \in X$ with $x \perp y$. Then there exists a unique A-additive mapping $g: X \rightarrow Y$ satisfying the $k$-orthogonal generalized quadratic functional equation (2.2) and

$$
\|f(x)-g(x)\| \leq \frac{c}{2|k|^{p}-2 k^{2}}\|x\|^{p}
$$

for all $x \in X$.

Proof. Letting $\rho(x, y)=c\left(\|x\|^{p}+\|y\|^{p}\right)$ for all $x, y \in X$ and choose $L=|k|^{2-p}<1$ in Theorem 2.7. Then we obtain this result.

Corollary 2.9. Let $X$ be an orthogonality module over a unital Banach algebra $A$ with the unit $e, Y$ be a real Banach module over $A, c>0, p, q \geq 0$ and with $p+q>2$. Suppose that $f: X \rightarrow Y$ is a mapping satisfying $f(0)=0$ and there exists a real number $k$ with $|k|>1$ such that

$$
\left\|D_{a}^{k} f(x, y)\right\| \leq c\|x\|^{p}\|y\|^{q}
$$

for all $a \in A_{1}$ and for all $x, y \in X$ with $x \perp y$. Then there exists a unique A-additive mapping $g: X \rightarrow Y$ satisfying the $k$-orthogonal generalized quadratic functional equation (2.2) and

$$
\|f(x)-g(x)\| \leq \frac{c}{2|k|^{p+q}-2 k^{2}}\|x\|^{p}
$$

for all $x \in X$.

Proof. Letting $\rho(x, y)=c\|x\|^{p}\|y\|^{q}$ for all $x, y \in X$ and choose $L=|k|^{2-p-q}<1$ in Theorem 2.7. Then we obtain this result. 
Corollary 2.10. Let $X$ be an orthogonality module over a unital Banach algebra $A$ with the unit $e, Y$ be a real Banach module over $A, c_{1}, c_{2}>0$ and $p>2$. Suppose that $f: X \rightarrow Y$ is a mapping satisfying $f(0)=0$ and there exists a real number $k$ with $|k|>1$ such that

$$
\left\|D_{a}^{k} f(x, y)\right\| \leq c_{1}\left(\|x\|^{p}+\|y\|^{p}\right)+c_{2}\|x\|^{\frac{p}{2}}\|y\|^{\frac{p}{2}}
$$

for all $a \in A_{1}$ and for all $x, y \in X$ with $x \perp y$. Then there exists a unique A-additive mapping $g: X \rightarrow Y$ satisfying the $k$-orthogonal generalized quadratic functional equation (2.2) and

$$
\|f(x)-g(x)\| \leq \frac{c_{1}+c_{2}}{\left(2|k|^{p}-2 k^{2}\right)}\|x\|^{p}
$$

for all $x \in X$.

Proof. Letting $\rho(x, y)=c_{1}\left(\|x\|^{p}+\|y\|^{p}\right)+c_{2}\|x\|^{\frac{p}{2}}\|y\|^{\frac{p}{2}}$ for all $x, y \in X$ and choose $L=|k|^{2-p}<1$ in Theorem 2.7. Then we obtain this result.

Acknowledgements: This work was support by Research Professional Development Project under the Science Achievement Scholarship of Thailand (SAST). The second author would like to thank the Thailand Research Fund and Office of the Higher Education Commission under grant no. MRG6180283 for financial support during the preparation of this manuscript.

\section{References}

[1] Rätz J., On orthogonally additive mappings, Aequ. Math., 1985, 28, 35-49

[2] Ulam S. M., Problems in Modern Mathematics, John Wiley \& Sons, New York, NY, USA, 1964

[3] Hyers D. H.,On the stability of the linear functional equation, Proc. Nat. Acad. Sci. U.S.A., 1941, 27, 222-224

[4] Aoki T., On the stability of the linear transformation in normed spaces, J. Math. Soc. Japan, 1950, 2,64-66

[5] Bourgin D. G., Classes of transformations and bordering transformations, Bull. Amer. Math. Soc., 1950, 57, 223-237

[6] Forti G. L., An existence and stability theorem for a class of functional equations, Stochastica, 1980, 4,23-30

[7] Rassias T. M., On the stability of the linear mapping in Banach spaces, Proc. Am. Math. Soc., 1978, 72, 297-300

[8] Gajda Z., On stability of additive mappings, Int. J. Math. Math. Sci., 1991, 14, 431-434

[9] Rassias T. M., Problem 16; 2.Report of the 27th International Symposium on Functional Equations, Aequ. Math., 1990, 39, 292-293

[10] Rassias T. M., On a modified Hyers-Ulam sequence, J. Math. Anal. Appl., 1991, 158, 106-113

[11] Ger R., Sikorska J., Stability of the orthogonal additivity, Bull. Pol. Acad. Sci., Math., 1995, 43, 143-151

[12] Skof F., Proprieta' locali e approssimazione di operatori, Rend. Semin. Mat. Fis. Milano, 1983, 53, 113-129

[13] Vajzović F., Über das Funktional $H$ mit der Eigenschaft: $(x, y)=0 \Rightarrow H(x+y)+H(x-y)=2 H(x)+2 H(y)$, Glas. Mat., 1967, 2, 73-81

[14] Towanlong W., Nakmahachalasint P., A quadratic functional equation and its generalized Hyers-Ulam Rassias stability, Thai J. Math. Special Issue (Annual Meeting in Mathematics), 2008, 2008, 85-91

[15] Diaz J., Margolis B., A fixed point theorem of the alternative for contractions on a generalized complete metric space, Bull. Amer. Math. Soc.,1968, 74, 305-309

[16] Park C., Cho Y. J., Lee J. R., Orthogonal stability of functional equations with the fixed point alternative, Adv. Difference Equ., 2012, 2012:173 